This is an electronic reprint of the original article. This reprint may differ from the original in pagination and typographic detail.

Author(s): Apaja, Vesa; Hyrkäs, Markku; Manninen, Matti

Title: $\quad$ Flat bands, Dirac cones, and atom dynamics in an optical lattice

Year: $\quad 2010$

Version:

Please cite the original version:

Apaja, V., Hyrkäs, M., \& Manninen, M. (2010). Flat bands, Dirac cones, and atom dynamics in an optical lattice. Physical Review A, 82(4), 41402.

https://doi.org/10.1103/PhysRevA.82.041402

All material supplied via JYX is protected by copyright and other intellectual property rights, and duplication or sale of all or part of any of the repository collections is not permitted, except that material may be duplicated by you for your research use or educational purposes in electronic or print form. You must obtain permission for any other use. Electronic or print copies may not be offered, whether for sale or otherwise to anyone who is not an authorised user. 


\title{
Flat bands, Dirac cones, and atom dynamics in an optical lattice
}

\author{
V. Apaja, M. Hyrkäs, and M. Manninen \\ Nanoscience Center, Department of Physics, University of Jyväskylä, FIN-40014 Jyväskylä, Finland
}

(Received 27 August 2010; published 13 October 2010)

\begin{abstract}
We study atoms trapped with a harmonic confinement in an optical lattice characterized by a flat band and Dirac cones. We show that such an optical lattice can be constructed which can be accurately described with the tight-binding or Hubbard models. In the case of fermions the release of the harmonic confinement removes fast atoms occupying the Dirac cones while those occupying the flat band remain immobile. Using exact diagonalization and dynamics we demonstrate that a similar strong occupation of the flat band does not happen in the bosonic case and furthermore that the mean-field model is not capable of describing the dynamics of the boson cloud.
\end{abstract}

Recent developments in manipulating cold atoms in optical lattices have opened new avenues for studying correlation and band structure effects in a controlled way [1-5]. One-, two-, and three-dimensional lattices with a variety of lattice structures have been proposed, including the kagome lattice [6-9], which was originally suggested by Syôzi [10] and studied intensively in the condensed-matter physics mainly due to its magnetic properties [11-14] and also thermodynamical properties [15]. In a simple tight-binding (TB) model the kagome lattice is characterized by a completely flat band, meaning that the energy is independent of the wave vector $\mathbf{k}$ and that electrons occupying this band are localized. The flat-band Hubbard model is a paradigm for ferromagnetism and has recently been reviewed by Tasaki [16].

The kagome lattice is one example of a whole class of two- (2D) and three-dimensional (3D) lattice structures which, in the TB model, produce a flat band $[17,18]$. In 2D these structures include square and hexagonal lattices and can have one or several flat bands as well as crossing points where two bands open as circular cones leading to Dirac fermions, as in graphene [19]. The Dirac fermions are also known to lead to interesting magnetic properties [20]. Allowing also the $p$ states in each lattice site to be occupied even more freedom to create flat bands and Dirac cones is obtained. Wu et al. [8] have studied the honeycomb (graphene) optical lattice with $p$ electrons and demonstrated the atom localization is due to the flat band of the system. Bercioux et al. [21] have shown that the $\mathcal{T}_{3}$ "dice" lattice has two Dirac cones and a flat band.

One of the simplest flat-band lattices is the edge-centered square lattice with three atoms in a unit cell [22], depicted in Fig. 1. In the TB model this lattice has three bands, the center band is flat and the upper and lower bands meet the flat band at the corners of the Brillouin zone where the Dirac cones open. This means that at the same energy the particle can have an infinite effective mass (flat band) or a zero effective mass (Dirac fermions). Recently Shen et al. showed that these massless Dirac fermions can exhibit perfect all-angle Klein tunneling [23]. This is the lattice of interest in the present work.

We first show that a laser field can be used to create a lattice which accurately produces a flat band and Dirac cones meeting at the same energy. The band structure is described with a TB model, or in the case of interacting atom, with a Hubbard model [2]. In the case of fermions with only one spin state (spinless fermions) the Hubbard model equals the noninteracting fermions since the Pauli exclusion principle prevents two similar fermions from occupying the same lattice point and on-site interaction has no effect. This provides us a simple way to study a dynamical problem; for example, what happens when a harmonic confinement, keeping the atoms in the central region of the lattice, is removed?

In the case of bosons the many-particle problem of the Hubbard model becomes more complicated. In this case we solve the problem with the Hubbard model for a small system of only three particles. However, this already demonstrates that (i) the boson system behaves differently than the fermion system and (ii) that frequently used mean-field models (e.g. Gross-Pitaevskii), where the boson system is described with only one single-particle wave function, fail to describe the dynamics correctly.

The results show that, when the harmonic confinement is removed, some of the atoms fly away fast while some remain stuck in the immobile flat-band states. We anticipate that this kind of experiment can be carried out in the near future using both bosonic and fermionic atoms.

The edge-centered square lattice with three atoms in a unit cell is illustrated in Fig. 1. We consider fist a simple TB model with only one state per lattice site, assume only nearest-neighbor hopping, and neglect the so-called differential overlap between neighbors. The band structure can be easily solved [24] by diagonalizing a $3 \times 3$ matrix for each $2 \mathrm{D}$ wave vector $\mathbf{q}=\left(q_{x}, q_{y}\right)$, resulting in energy levels

$$
\begin{gathered}
\epsilon_{1}(\mathbf{q})=-t \sqrt{4+2 \cos \left(q_{x} a\right)+2 \cos \left(q_{y} a\right)}, \\
\epsilon_{2}(\mathbf{q})=0, \\
\epsilon_{3}(\mathbf{q})=t \sqrt{4+2 \cos \left(q_{x} a\right)+2 \cos \left(q_{y} a\right)},
\end{gathered}
$$

where $t$ is the strength of the hopping integral between nearest neighbors and $a$ the lattice constant. The corresponding wave vectors $v_{i}=\left(v_{\mathrm{c}}, v_{\mathrm{e} 1}, v_{\mathrm{e} 2}\right)$, where the three components refer to the corner and edge sites in the unit cell, are $\left(s=\sqrt{4+e^{i q_{x} a}+e^{-i q_{x} a}+e^{i q_{y} a}+e^{-i q_{y} a}}\right)$

$$
\begin{gathered}
v_{1}(\mathbf{q})=\left(-s, 1+e^{-i q_{y} a}, 1+e^{-i q_{x} a}\right), \\
v_{2}(\mathbf{q})=\left(0,1+e^{i q_{x} a}, 1+e^{i q_{y} a}\right), \\
v_{3}(\mathbf{q})=\left(s, 1+e^{-i q_{y} a}, 1+e^{-i q_{x} a}\right) .
\end{gathered}
$$



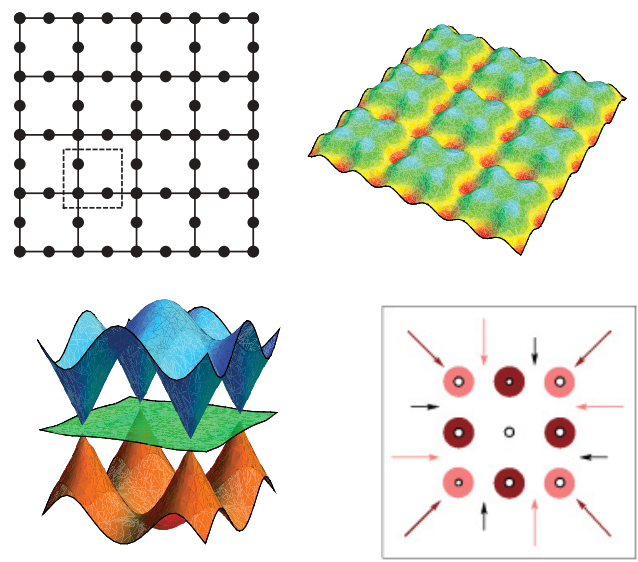

FIG. 1. (Color online) Top left: Edge-centered square lattice and its unit cell shown with a dashed line. Top right: Optimized potential, Eq. (3), with equally deep minima. Bottom left: Three lowest bands corresponding to the potential of Eq. (3). The cusps are at the corners of the Brillouin zone. Bottom right: Arrows show the suggested directions of the laser fields causing potentials like $\cos ^{2} x$, $\cos ^{2}(x+y)$, and $\cos ^{2}(2 x)$, corresponding to the two steep minima and one shallow minimum.

The energy bands consist of a flat band at zero energy and two bands symmetrically below and above the flat band. All bands meet at points at the corners of the Brillouin zone, $q_{x}=q_{y}=\pi / a$. The band structure is shown in Fig. 1. The wave function of the flat band has zero amplitude at the corner point of the square lattice. This is the reason for the flatness: The particles in these states do not hop to the neighboring sites and can be viewed as localized. The effective band mass of the particles is thus infinite.

Close to the zero energy the two other bands form "Dirac cones," and the velocity of the particles is independent of the energy, $\epsilon_{1,3}\left(k_{x}-\pi / a, k_{y}-\pi / a\right) \approx \mp t a \sqrt{k_{x}^{2}+k_{y}^{2}} / 4=$ $\mp t a k / 4$, where $k$ is the distance from the point of the cusp. The linear dispersion relation means a zero effective mass. Interestingly, in this lattice we have at the same energy region particles with zero effective mass and infinite effective mass. Note that the cones appear to be symmetric to rather high energies, indicating that most of the particles in the lowest and uppermost bands have nearly equal velocities.

Next we will consider how such a simple TB lattice could be constructed for cold atoms in an optical lattice. We do not go into the details of the interactions between the atoms and the laser fields [1-3], but simply assume that the effective potential caused by the standing electromagnetic waves can be described with trigonometric functions $\left[\cos ^{2}(x)\right.$ or equivalently $\cos (2 x)]$. Clearly a square lattice can be made as $-\cos ^{2}(a x / \pi)-\cos ^{2}(a y / \pi)$, having minima at lattice sites $R=(n a, m a)$. Minima at the edge centers can be constructed with functions $\cos ^{2}[a(x+y) / 2]+\cos ^{2}[a(x-y) / 2]$. However, with a linear combination of these two it is not possible to construct proper potential barriers between corner end edge sites and an additional shorter wavelength function of $-\cos ^{2}(2 a x / \pi)-\cos ^{2}(2 a y / \pi)$ has to be added. The potential optimized to give the band structure similar to that of the TB band structure is

$$
\begin{aligned}
V_{\mathrm{OL}}(x, y)= & V_{0}\left\{-0.9\left[\cos ^{2}(a x / \pi)+\cos ^{2}(a y / \pi)\right]\right. \\
& +0.496\left\{\cos ^{2}[a(x+y) / 2]+\cos ^{2}[a(x-y) / 2]\right\} \\
& \left.+\left[\cos ^{2}(2 a x / \pi)+\cos ^{2}(2 a y / \pi)\right]\right\},
\end{aligned}
$$

where $V_{0}$ gives the energy scale (see the following paragraph). The resulting potential (nine unit cells) is shown in Fig. 1. The band structure corresponding to the potential (3) was computed using a $26 \times 26$ real space grid in the square unit cell with lattice constant $a$. The $\nabla^{2}$ differential operator (in the Schrödinger equation) was approximated with the nearest-neighbor grid points. Periodic boundary conditions and Bloch's theorem were employed for each $k$ point. The numerical parameters in Eq. (3) were determined by minimizing the width of the flat band using $100 \times 100 k$ points in the Brillouin zone. Figure 1 shows the three lowest bands computed for the potential (3).

The parameter $V_{0}$ in Eq. (3) is related to the mass of the atoms $m$ and the lattice constant $a$ as $V_{0} \approx 497 \hbar^{2} /\left(2 m a^{2}\right)$ (where the numerical factor 497 comes from fitting of the band structure to that of the TB model).

The overall width of the three lowest bands is $7.65 \hbar^{2} /\left(2 m a^{2}\right)$ while the width of the flat band is only $0.11 \hbar^{2} /\left(2 m a^{2}\right)$, meaning that the dispersion of the flat band is only $1.5 \%$ of the total width of the three bands. The potential of Eq. (3) has, of course, an infinite number of other bands, but there is a huge gap of $140 \hbar^{2} /\left(2 m a^{2}\right)$ between the three lowest bands and the next band. With this example we have demonstrated that a proper combination of cosine potentials can produce a band structure which, with a high accuracy, gives energy bands similar to those of the simple TB model. Figure 1 shows schematically the laser arrangement needed for making such an optical lattice.

We assume that the atoms trapped by the optical lattice have contact with a repulsive short-range potential and we assume the interaction is so strong that only one atom is allowed in each lattice site. The many-particle problem then reduces to the Hubbard Hamiltonian. We first consider fermionic atoms with only one spin state (spinless fermions). In this case the manyparticle problem is noninteracting, since the Pauli exclusion principle already prevents two atoms from occupying the same site.

In the actual experiments the optical lattice is superimposed on a harmonic confinement which localizes the particles (with repulsive interaction) in the central region of the lattice. By putting enough atoms in the lattice, the harmonic confinement guarantees that in the central region the occupation of each lattice site is nearly one. This means that all three bands are filled at the central region of the harmonic confinement.

Our interest is to see what happens when the harmonic confinement is removed but the optical lattice is kept in place. This dynamical problem is easily solved for $N$ spinless fermions. The procedure is as follows: We solve the TB model in a large finite lattice with a harmonic confinement and fill the lowest $N$ single-particle states. This is the fermionic ground state $\Psi_{0}(0)$ at time zero. Next we remove the harmonic confinement and expand the states with the confinement in terms of the states $\Psi^{0}$ calculated without the confinement. The 


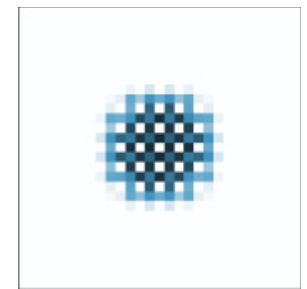

(a) time $=0$

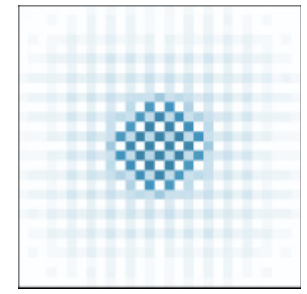

(c) time $=5$

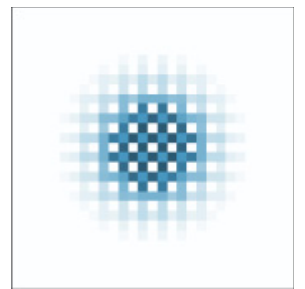

(b) time $=2.5$

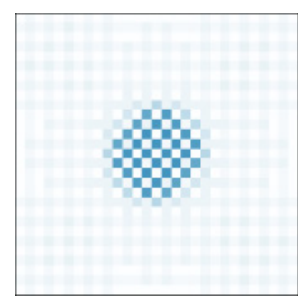

(d) time $=7.5$
FIG. 2. (Color online) Time evolution of 50 fermionic atoms when the harmonic confinement is removed. Darker color refers to higher density, ranging from 0 to 1 . (a) shows the initial state with the harmonic confinement of strength $U_{\mathrm{h}}=0.4$ (in units of $\hbar^{2} / 2 m a^{2}$ ), and (d) the 'final' state when atoms trapped by the flat band stay localized while other atoms have flown away.

time dependence follows then from the time dependencies of each individual state as

$$
\Psi_{0}(t)=\sum_{j} C_{j} e^{-i E_{j} t / \hbar} \Psi_{j}^{0}(0),
$$

where $E_{j}$ is the energy of the many-particle state $j$. In the case of spinless fermions where each state is a Slater determinant of single-particle states the time dependence further reduces to the time dependencies of the single-particle states.

In practice we solved up to 100 atoms in a lattice of $25 \times 25$ unit cells. We use TB units with the hopping parameter $t=1$ and the nearest-neighbor distance $b=a / 2=1$. The harmonic confinement adds to diagonal terms to the TB Hamiltonian matrix: Placing the center of the finite lattice at the origin, the harmonic confinement changes the energy level of a lattice site by $U_{\mathrm{h}} r^{2}$, where $U_{\mathrm{h}}$ is the strength of the harmonic confinement and $r$ the distance of the lattice site from the origin (in units of the lattice constant). Figure 2 shows an example of such a simulation. The Harmonic confinement localizes the atom cloud at the center of the finite lattice. The maximum density is at the edge sites of the unit cell with occupation about 0.8 , while at the corner sites it is about 0.6. When the harmonic confinement is removed, the cloud fast expands [Figs. 2(b) and 2(c)], but some of the atoms stay trapped at the edge sites [Fig. 2(d)], forming a lattice of localized atoms. The occupation of the edge states remains close to 0.5 . The TB band is exactly flat, so the atoms that are trapped in Fig. 2(d) stay trapped forever.

The explanation of the observed dynamics is a direct result of the single-particle properties of noninteracting fermions: Those occupying the bands 1 and 3 are mobile and fly quickly away (at the Dirac cones even with the same velocity), while atoms occupying the flat band are immobile and stay in place. Notice that the corner states of the unit cell are emptied since the flat band does not have any amplitude in these sites.
The bosonic many-particle problem is usually simpler than the fermionic case due to the symmetry of the wave function. In our case, however, this is not the case. Even if we assume an infinitely strong contact interaction, the bosonic case remains a true many-particle problem, although the boson and fermion problems are related [25]. A common approach is to use a mean-field model for the Hubbard Hamiltonian (this is closely related to the Gross-Pitaevskii [26,27] model): The interaction term $U\left(\hat{n}_{i}-1\right) \hat{n}_{i}$ is replaced with $U\left(\left\langle n_{i}\right\rangle-1\right) \hat{n}_{i}$ (where $\hat{n}_{i}$ is the occupation number operator). The first approximation is to assume that all the bosons occupy the same quantum state determined by the external confinement.

Making the interaction $U$ very large, we prevent the occupation of any lattice site to be much larger than one. In the harmonic confinement we can then get the initial atom density to be qualitatively similar to that of the fermionic case and one would expect that by expanding the self-consistent bosonic ground state in terms of the single-particle band states also the flat band would be markedly occupied. However, this is not the case. The occupation of the flat-band state remains less than $2 \%$ even when the interaction strength $U$ is extrapolated to infinity. This means that when the confinement was removed, practically all the atoms of the cloud escape quickly.

In order to understand if the boson system behaves truly differently than the fermion system, or if it is the meanfield approach which is not appropriate for our system, we performed a small-scale exact diagonalization of the Hubbard Hamiltonian. The test size was $3 \times 3$ unit cells with periodic boundary condition, that is, 27 sites altogether as shown in Fig. 3. We assumed infinitely strong repulsive interaction between the atoms $(U=\infty)$ which reduced the boson basis to states having at most one atom in a lattice site. Initially the atoms were localized in a small region with strong attractive potentials in five adjacent sites as shown in Fig. 3. By diagonalizing the Hubbard Hamiltonian for three bosons (and fermions) with and without the attractive potentials at five sites, the time evolution could be solved exactly using Eq. (4).

The time evolution is shown in Fig. 3 for bosons and fermions. In the case of fermions the results are qualitatively similar to those of the larger systems described previously.
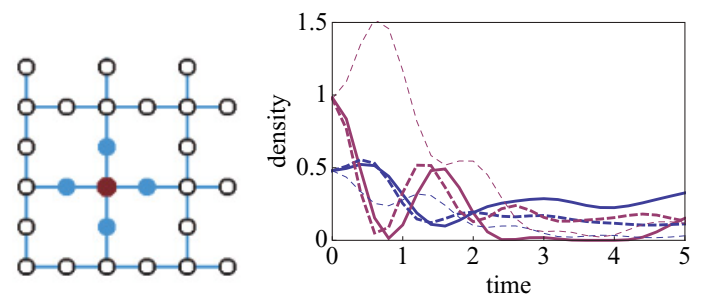

FIG. 3. (Color online) Left: Finite lattice used, with periodic boundary conditions, for solving the Hubbard model for three bosons or fermions. The initial potential at the cites denoted by red (dark gray) and blue (light gray) were $-20 t$ and $-5 t$, respectively. Right: Time dependence of the occupation of red (dark gray) and blue (light gray) sites after the potential of these sites were put to zero. Solid lines are the results for fermions and the thick dashed lines are the results for bosons. The thin dashed lines are results for a single-particle state with the same initial density as the exact boson result, indicating that the mean-field solution fails in this case. The time is in units of $\hbar / t$. 
After a short time the atom density at the corner site decreases to nearly zero while at the edge sites it stays quite large, averaging to a value of about 0.3 after initial oscillations. In the case of bosons the initial time dependence is very similar, but soon both center and edge sites average to the same value of about 0.1 , which is close to the average filling of the lattice $3 / 27=1 / 9$. Figure 3 also shows the time evolution of a single state which initially had the same density distribution as the bosonic case. Clearly, this time evolution is qualitatively different indicating that a mean-field (single state) approximation for the bosonic system cannot describe correctly the time evolution in the present case.

In conclusion, we have shown that by using cosine functions a rather simple lattice can be constructed, which has a flat band and Dirac cones meeting at the same energy. The three lowest bands of the lattice can be accurately described with a simple tight-binding model with only one state per site. By confining atoms with a harmonic confinement in such a lattice, fermion atoms stay trapped in the flat-band states even when the harmonic confinement is removed.

The case of bosons is more complicated and requires further study. Using the Hubbard model with infinitely strong interaction $(U \rightarrow \infty)$ we demonstrated that the trapping of the bosons is not as strong as in the case of fermions. A similar result was obtained with the mean-field approximation for bosons, although comparison to the exact solution of the Hubbard model indicated that the mean-field model fails to describe the boson dynamic correctly in the system studied.
[1] I. Bloch, Nat. Phys. 1, 23 (2005).

[2] A. C. Leggett, Quantum Liquids: Bose Condensation and Cooper Pairing in Condensed Matter Systems (Oxford University Press, London, 2006).

[3] C. J. Pethick and H. Smith, Bose-Einstein Condensation in Dilute Gases, 2nd ed. (Cambridge University Press, Cambridge, UK, 2008).

[4] I. Bloch, J. Dalibard, and W. Zwerger, Rev. Mod. Phys. 80, 885 (2008).

[5] S. López-Aguayo, Y. V. Kartashov, V. A. Vysloukh, and L. Torner, Phys. Rev. Lett. 105, 013902 (2010).

[6] L. Santos, M. A. Baranov, J. I. Cirac, H.-U. Everts, H. Fehrmann, and M. Lewenstein, Phys. Rev. Lett. 93, 030601 (2004).

[7] B. Damski, H. Fehrmann, H.-U. Everts, M. A. Baranov, L. Santos, and M. Lewenstein, Phys. Rev. A 72, 053612 (2005).

[8] C. Wu, D. Bergman, L. Balents, and S. Das Sarma, Phys. Rev. Lett. 99, 070401 (2007).

[9] J. Ruostekoski, Phys. Rev. Lett. 103, 080406 (2009).

[10] I. Syôzi, Prog. Theor. Phys. 6, 306 (1951).

[11] S. Sachdev, Phys. Rev. B 45, 12377 (1992).

[12] J. Greedan, J. Mater. Chem. 11, 37 (2001).

[13] T. Kimura, H. Tamura, K. Shiraishi, and H. Takayanagi, Phys. Rev. B 65, 081307 (2002).

[14] L. Balents, Nature (London) 464, 199 (2010).
[15] O. Derzhko, J. Richter, A. Honecker, M. Maksymenko, and R. Moessner, Phys. Rev. B 81, 014421 (2010).

[16] H. Tasaki, Eur. Phys. J. B 64, 365 (2008).

[17] S. Deng, A. Simon, and J. Köhler, J. Solid State Chem. 176, 412 (2003).

[18] S. Miyahara, K. Kubo, H. Ono, Y. Shimomura, and N. Furukawa, J. Phys. Soc. Jpn. 74, 1918 (2005).

[19] A. C. Neto, F. Guinea, N. Peres, K. Novoselov, and A. Geim, Rev. Mod. Phys. 81, 109 (2009).

[20] Z. Meng, T. Lang, S. Wessel, F. Assaad, and A. Muramatsy, Nature (London) 464, 847 (2010).

[21] D. Bercioux, D. F. Urban, H. Grabert, and W. Häusler, Phys. Rev. A 80, 063603 (2009).

[22] H. Aoki, M. Ando, and H. Matsumura, Phys. Rev. B 54, R17296 (1996).

[23] R. Shen, L. B. Shao, Baigeng Wang, and D. Y. Xing, Phys. Rev. B 81, 041410(R) (2010).

[24] N. Ashcroft and N. Mermin, Solid State Physics (Saunders, Philadelphia, 1976).

[25] S. Ouvry and A. Polychronakos, J. Phys. A: Math. Theor. 42, 275302 (2009).

[26] E. P. Gross, Nuovo Cimento 20, 454 (1961).

[27] L. P. Pitaevskii, Zh. Eksp. Teor. Fiz. 40, 646 (1961) [Sov. Phys. JETP 13, 451 (1961)]. 